\title{
Early Fire Smoke Image Segmentation in a Complex Large Space
}

\author{
Hu Yan ${ }^{1,2, *}$, Wang Huiqin ${ }^{1,2}$, Zhao Qian ${ }^{3}$ and Lu Ying ${ }^{2}$ \\ ${ }^{I}$ School of Management, Xi'an University of Architecture and Technology, Xi'an, Shaanxi, 710055, China; ${ }^{2}$ School of \\ Information and Control Engineering, Xi'an University of Architecture and Technology, Xi'an, Shaanxi, 710055, China; \\ ${ }^{3} X i$ 'an Modern Control Technology Research Institute, Xi'an, Shaanxi, 710065, China
}

\begin{abstract}
Aiming at the problem that the fire smoke image segmentation algorithm cannot obtain integral smoke information, the adaptive background updating smoke foreground object extraction algorithm based on block segmentation is proposed. According to the characteristics that the smoke internal pixels continuously roll in the heat of the driver and the pixel concentration from near the fire source to edge decreases in turn, the method for different blocks using different difference threshold is adopted to ensure the integrity of the extracted foreground target. Under the difference between the current frame and the background frame, block difference thresholds are updated to let the differential threshold adjustment with continuous adaptive monitoring. In the new weighted average updated background image corrected by the original background image, the interference suspicious target edge pixels are removed. Simulation experimental results show that the method is able to extract more complete information of smoke in a suspected area of the edge, and eliminate the interference of light and pedestrian in complex space.
\end{abstract}

Keywords: Background updating, blocking, complex large space, connected domain, smoke image segmentation.

\section{INTRODUCTION}

The smoke produced in the smoldering stage is a very significant character of early fire. Because the smoke affected by plume effect is not sheltered by an obstacle, the analysis and recognition of smoke image can help achieve early realization of the fire warning [1]. Accurate segmentation of smoke image is the premise and foundation for feature extraction and target recognition. However, compared with the flame image, smoke image features, such as color, gradient, etc. are not clear, and more vulnerable to the outside interferences. Those factors makes the smoke image segmentation relatively complex. The improved HIS mode is proposed to get smoke area, but the background color will influence the smoke color judgment [2]. The fog image is transformed by dual tree complex wavelet. The hidden Markov tree model is structured to express the smoke texture, and the smoke is detected by support vector machine. The disadvantage is the high false rate [3]. Using the characteristics of wavelet transform spatial frequency, smoke texture enhancement image is achieved by weighted average processing of the image after wavelet transform coefficients decomposition into multilayer. Then, using the differential box counting method to calculate the numerical traversing of the image, to get each pixel fractal dimension, smoke segmentation image is obtained by threshold method. When the texture information of an image is not rich, this method can easily produce the over segmentation [4]. Single Gauss background difference method is presented to obtain the foreground object. Because the difference threshold is fixed, it cannot meet the demand of automatic fire smoke detection [5].

\footnotetext{
*Address correspondence to this author at the School of Information and Control Engineering, Xi'an University of Architecture and Technology, Xi'an, Shaanxi, 710055, China; Tel: 13772162942; E-mail: huyan.nancy@163.com
}

In the early fire smoke image detection, the difference in smoke concentration leads to different extents of shading for the background. So, using the characteristics of early smoke, blocking technology will be applied to image segmentation to improve smoke image segmentation effect. And the method has a certain practicality.

\section{SMOKE IMAGE SEGMENTATION MODEL}

Moving target detection technology [6] extracts foreground object through analyzing image motion information. Background difference method and frame difference method are good real-time and favored by simple calculation. Because of unique characteristics of smoke image, if these methods are directly applied to the smoke image segmentation, there exist the following problems:

1) Owing to smoke image color non-uniform, the background difference method is sensitive to illumination changes. That fixed difference threshold is too large or too small is prone to over segmentation or leakage segmentation.

2) Smoke image motion is slow in consecutive frames. Frame difference method produces larger voids. Extraction of the target is not complete.

Smoke can be divided into smoldering smoke and fire smoke [7]. Early fire recognition focuses on the smoldering smoke. Smoldering smoke image color is given priority to white, grey and white. Smoke keeps churning in the heat. The smoke concentration near the fire point is greater than the edge. Adopting the block technology, the different blocks with different difference threshold, when suspected smoke number of pixels in block meets the preset condition, the block is smoke area, otherwise regarded as disturbance. The specific steps are as follows: 
Step 1: Block segmentation. $I(x, y)$ is the current frame at $t$ moment. $B(x, y)$ is the background frame. The two frames size is $M \times N$. Every block size of the frame is $L \times L$. Extraction of suspicious targets in block units not only can improve the efficiency of detection, but also reduce noise interference. ${ }^{i}$ is a cyclic variable, which represents the number of image blocks. Each block is handled as follows:

$$
D_{i,}(x, y)= \begin{cases}1, & \left|I_{s}(x, y)-B_{i,}(x, y)\right|>T_{t, i},\left(i-i-1,2,3, \cdots,\lfloor M / L\rfloor \times N_{-} / L_{-}\right) \\ 0, & \text { others }\end{cases}
$$

$I_{t, i}(x, y), B_{t, i}(x, y), D_{t, i}(x, y)$ and $T_{t, i}$ respectively express the current frame, background frame, front two difference image, and block difference threshold for $i$ block of $(x, y)$ pixel at the $t$ time.

Num is pixel's number of satisfying (1) in the $i$ block. If Num is greater than $0.75 \times(L \times L)$, the block is considered suspicious smoke object. If the pixels of this object are intercalated, it is 1 , otherwise 0 .

Step 2: Judgment of connected domain blocks [8]. Smoke is a slow process of change from appearance to perdition. When a suspicious object area is larger than a certain threshold, it is probably because mutation caused by illumination leads to the area to relatively large, even close to full frame image. In addition, a little suspicious objects may also be extracted by the effects of noise. So, the connected domain area is counted whether or not this is smoke.

$$
\text { Area }_{-} \text {mum }_{R}=\sum_{(x, y) \in R_{j}} D(x, y)
$$

Area_num ${ }_{R}$ is a parameter to express the area of connected domain $R_{j}(j=1,2, \ldots, m)$. When the parameter Area_num ${ }_{R} /(M \times N)$ is greater than $4 \%$ and less than $60 \%, R_{j}$ is smoke.

Step 3: Processing of morphology [9]. The use of open operation can eliminate scatter and burr smooth effect on the image. Closed operation can fill the void, by bridging the crack. The two-image processing cannot change the location and shape of the original image. They are defined as follows:

$$
\begin{aligned}
& X \nwarrow S=(X \Theta S) \oplus S \\
& X \bullet S=(X \oplus S) \Theta S
\end{aligned}
$$

Step 4: Differential threshold updating. The differential threshold of moving object detection based on background difference could influence the effect of target detection. Because of smoke reunion characteristic [10], the block based differential threshold updating can guarantee within a certain time interval that the smoke can be accumulated with enough differences and formed potentially suspicious target formation. If the current block is detected as a suspicious object, it shows that this block differential threshold is proper. Otherwise, it shows that this block differential threshold is too large. It therefore, needs to be updated. Differential degree between current frame and background frame is used as the updating reference. The mean differential threshold of all pixels is used as the block final differential threshold.

$$
T_{t+1, i}=\left\{\begin{aligned}
\frac{1}{L \times L} \sum_{(x, y) \in L \times L} \alpha \times T_{t, i}+(1-\alpha)\left|I_{t, i}(x, y)-B_{t, i}(x, y)\right|, & \\
& \text { if block } k_{t, i}(x, y)=0 \\
T_{t, i}, & \text { if } b \operatorname{lock}_{t, i}(x, y)=1
\end{aligned}\right.
$$

$\alpha$ is a scale factor, which generally takes 0.05 .

Step 5: Background image updating. In practical situations, appearance of fire smoke circumstances are less. In order to improve the detection speed of the algorithm, the background image updating is divided into global updating and local updating. If the current frame is not extracted into suspicious objects, background image is globally updated with the current frame. But thanks to the existence of noise, a small amount of suspicious objects could inevitably be extracted. Therefore, when the number of pixels in the suspicious object is less than the threshold, they are not smoke objects, and the current frame is used as a new background image. The emergence of fire smoke is slow growth change process. It may not start to occupy most of the regional image. So, when the number of suspicious object pixel is over the image size of $70 \%$, this is probably because of sudden illumination changes, such as switching lights, led to the abnormal foreground extraction, then to the background of global updating. Following the above situation, the background image is locally updated.

$B_{t+1, i}(x, y)= \begin{cases}\alpha \times B_{t, i}(x, y)+(1-\alpha) I_{t, i}(x, y), & \text { if } \text { block }_{t, i}(x, y)=0 \\ B_{t, i}(x, y) & , \text { if } \text { block }_{t, i}(x, y)=1\end{cases}$

block $_{t, i}(x, y)$ is $i$ block pixel coordinate value at $t$ time.

Smoke image segmentation algorithm flow chart is shown in Fig. (1).

Two-smoke images are segmented as shown in Fig. (2). by using Fig. (1). respectively.

The proposed algorithm can extract more complete smoke foreground target, maintain the original smoke image irregular edge information, and eliminate the interference of similar color with smoke, see Fig. (2b). The time of segmentation algorithm is less than $1.5 \mathrm{~s}$, meeting the real-time requirements of the system. But, updating background image still retains a small smoke around the target, see Fig. (2c). When this background image is used as the new background image again to get foreground object, it is easier to cause that the target edge information is not complete.

\section{CORRECTION OF THE BACKGROUND IMAGE UPDATING}

Because of the characteristic of the smoke concentration that it gradually becomes weaker from the interior to the edge, the color in the edge is dark similar to the background. The updating background image still retains a small smoke around the target, see Fig. (2c) by formula (6). It is very unfavorable for follow-up of the suspicious targets detection. 


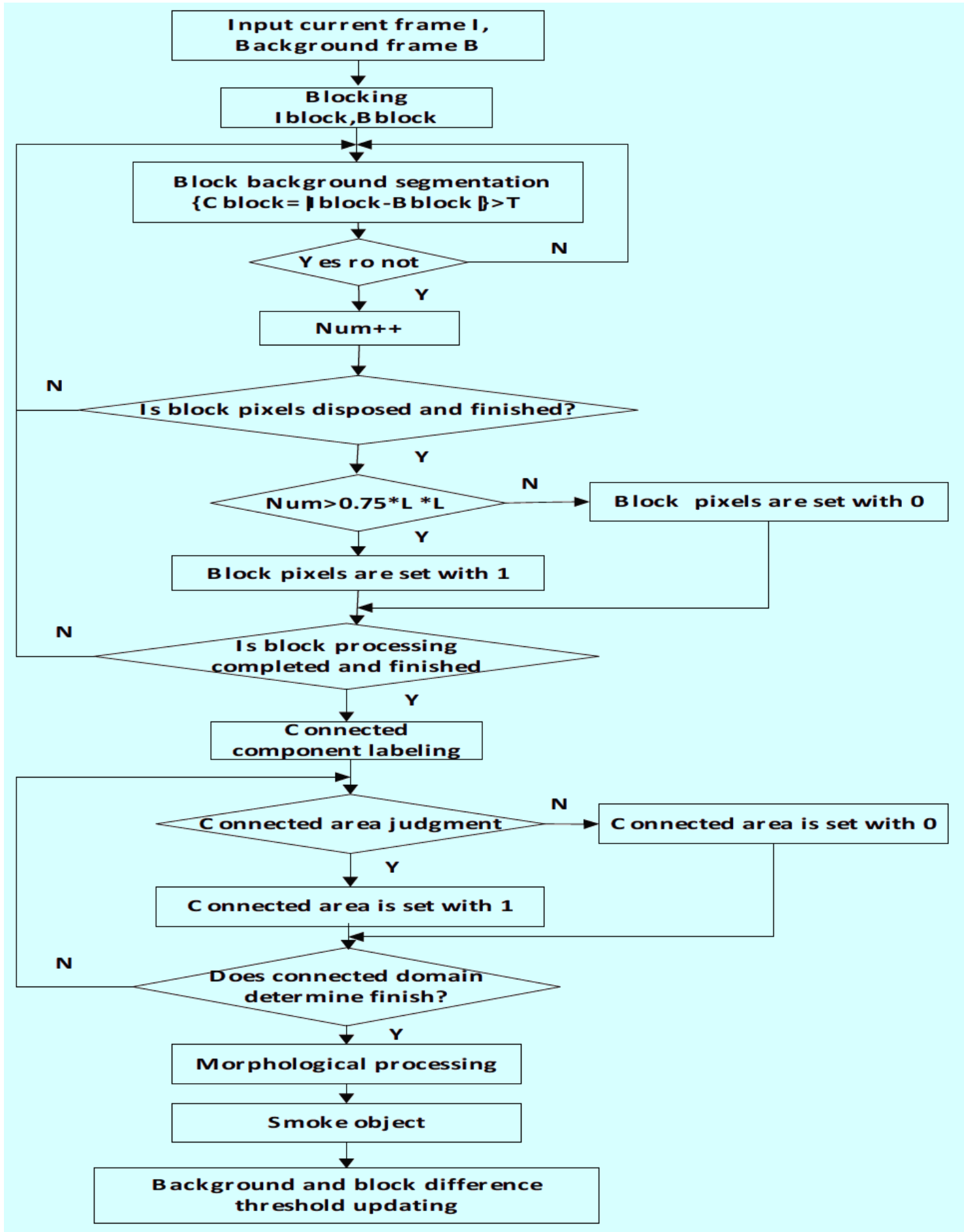

Fig. (1). Flow chart of smoke image segmentation algorithm.

So, the formula (6) is modified by the original background image to get equation 7 . The simulation result is shown in Fig. (3).

$$
B_{t+1, i}(x, y)^{\prime}=\beta \times B_{t, i}(x, y)+(1-\beta) B_{t+1, i}(x, y)
$$

It can be seen that there are traces of small smoke around the target as shown in Fig. (2c). They are smoothed well by using formula (7) so that the new background image is more close to the true background.

\section{SIMULATION EXPERIMENTS AND RESULTS ANALYSIS}

In order to verify the performance of the segmentation algorithm, four videos are examined respectively. A part of videos are shot by authors' research group including smoke and interference videos. Another part of videos are from http://signal.ee.bilkent.edu.tr/VisiFire. The segmentation results are shown in Fig. (4). 


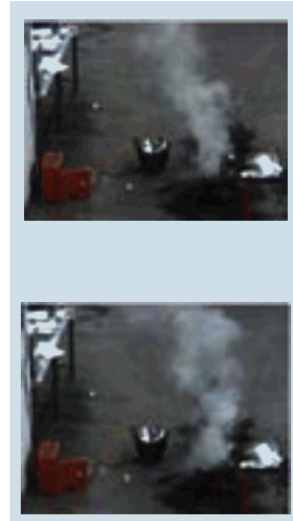

(a) Original images
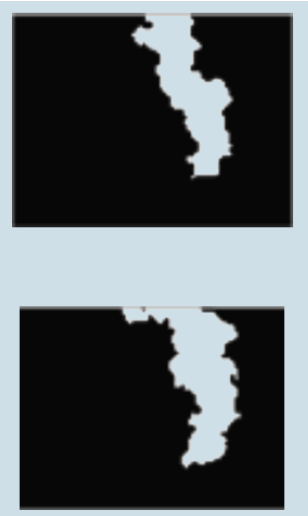

(b) Proposed algorithm
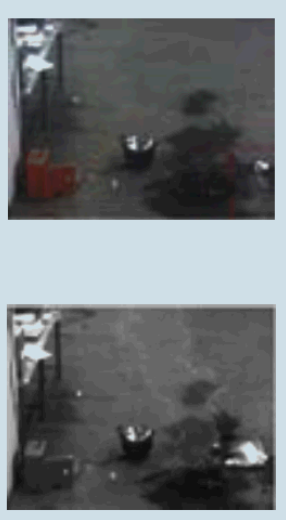

(c) Original background and updated background image

Fig. (2). Smoke image segmentation and background updating based on block.

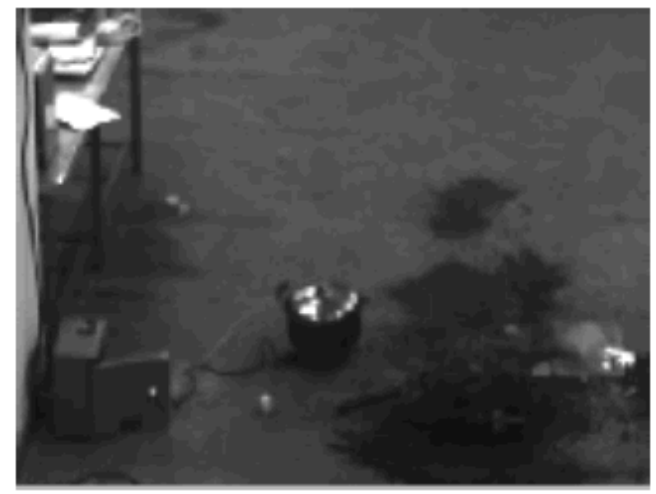

Fig. (3). Correction for Fig. (2c) $(\beta=0.7)$.

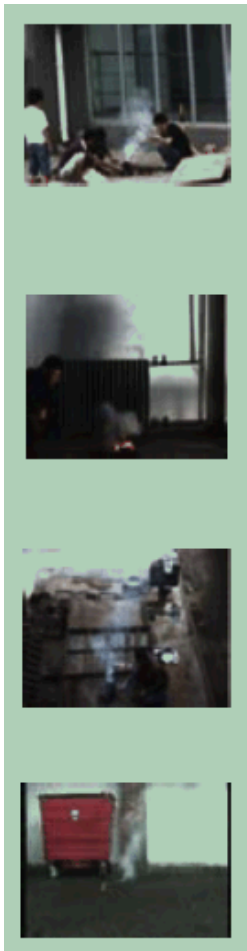

(a) Smoke images
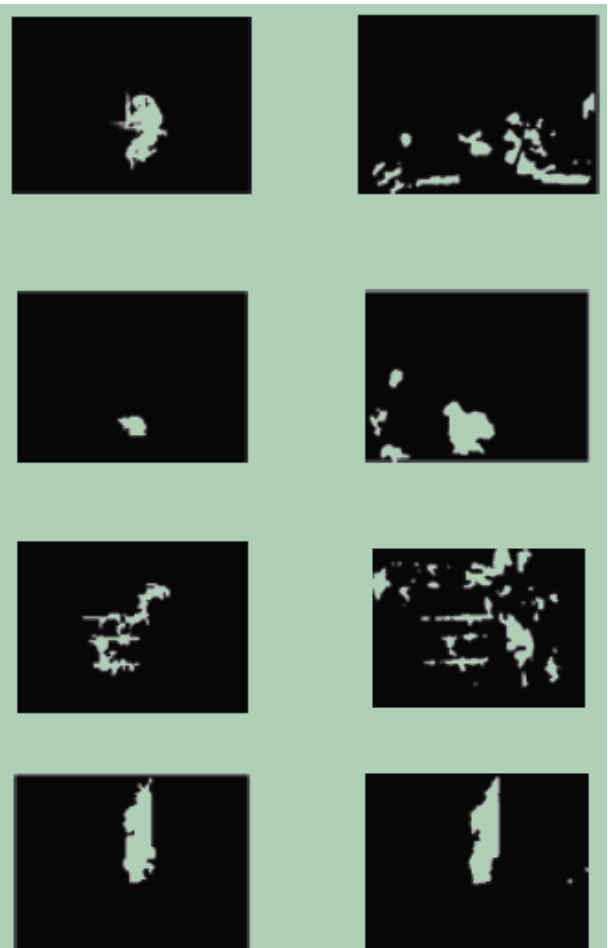

(b) Proposed algorithm

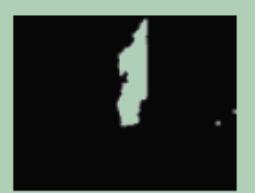

(c) The background difference method

Fig. (4). Smoke images and the segmentation results. 
Compared with the background difference method, the proposed algorithm can extract more complete smoke target, and eliminate interferences, for example pedestrian, complex background, light and reflected light. However, indoor smoke image segmentation effect of background difference method is better than that of outdoor. This is mainly because of interferences, such as light changes, reflecting light in outdoor complex space. Those interferences will result in the excessive object segmentation region, which will affect the accuracy of target recognition.

\section{CONCLUSION}

Image segmentation is the premise and foundation of pattern recognition. It can reduce the amount of data to be processed, and enhance the real-time of the algorithm. At present, the segmentation method of fire smoke image commonly use fixed threshold method which is prone to over segmentation or leakage segmentation. And this segmentation method gave incomplete information of the edge, which is not conducive to the suspected target feature extraction. Therefore, smoke image segmentation algorithm based on blocking and adaptive background updating is proposed. The method uses image characteristics in the spatial domain changes. Different blocks with different difference thresholds can extract more complete suspected smoke target. And real-time differential threshold is updated. The background image updated by weighting is revised using the original background image to ensure that the new background image can be an even more true reflection of the scene change. Simulation results show that this method can extract most of the suspected smoke image in a complex space, the edge information is relatively complete, and is strongly interference resistant.

\section{CONFLICT OF INTEREST}

The authors confirm that this article content has no conflict of interest.

\section{ACKNOWLEDGEMENTS}

Sincere thanks for the teacher Wang for his careful guidance in the practice of scientific research and paper writing. At the same time, I would also like to thank the members of the research team for their help and support.

Foundation: The special scientific research plan project of Shaanxi Province Education Office (14JK1438), and The major science and Technology achievement innovation fund of Xi'an University of Architecture and Technology (ZC1103) are acknowledged.

\section{REFERENCES}

[1] T. Chen, Y. Yin, S. Huang, and Y. T. Ye, "The smoke detection for early fire alarming system based on video processing," $I I H$ MSP'06, Pasadena, California, USA, 2006, pp. 427-430.

[2] D. Krstinic, D. Stipanicev, and T. Jakovcevic, "Histogram-based smoke segmentation in forest fire detection system," Information Technology and Control, vol. 38, pp. 237-244, 2009.

[3] R. J. Ferrari, H. Zhang, and C. R. Kube, "Real-time detection of steam in video image," Pattern Recognition, vol. 40, pp. 1148$1159,2007$.

[4] Y. Huang, and Y. Lv, "Early fire smoke image segmentation based on enhancement of frequency field and differential box-Counting fractal dimension," Laser Journal, vol. 35, pp. 66-69, 2014.

[5] J. Yang, F. Chen, and W. Zhang, "Visual-based smoke detection using support vector machine," ICNC, Jinan, China, 2008, pp. 301305.

[6] D. Han, and B. Lee, "Flame and smoke detection method for earlytime detection of a tunnel fire," Fire Safety Journal, vol. 44, pp. 951-961, 2009.

[7] C. Yu, J. Fang, J. Wang, and Y. Zhang, "Video fire smoke detection using motion and color features," Fire Technology, vol. 46, pp. 651-663, 2010.

[8] F. Zhao, Z. Lu, Z. Zhang, and H. Lu, "A hardware acceleration based algorithm for real-time binary image connected-component labeling," Journal of Electronics \& Information Technology, vol. 33, pp. 1069-1075, 2011.

[9] M. M. Thouraya, B. Malika, and B. Bachir, "Mathematical morphology for TOFD image analysis and automatic crack detection," Ultrasonics, vol. 54, pp. 1642-1648, 2014.

[10] A. Rafiee, R. Tavakoli, R. Dianat, and S. Abbaspour, "Fire and smoke detection using wavelet analysis and disorder characteristics," ICCRD $3^{\text {rd }}$ International Conference on Computer Research and Development, vol. 3, 2011, pp. 262-265.

Received: December 17, 2014

Revised: January 25, 2015

Accepted: May 14, 2015

(C) Yan et al.; Licensee Bentham Open.

This is an open access article licensed under the terms of the Creative Commons Attribution Non-Commercial License (http://creativecommons.org/licenses/by-nc/4.0/) which permits unrestricted, non-commercial use, distribution and reproduction in any medium, provided the work is properly cited. 